\title{
Sculpturing Squeezed States to Get Highly Excited Fock States
}

\author{
Paula B. Monteiro, B. Baseia, \\ Instituto de Física, Universidade Federal de Goiás, 74.001-970, Goiânia (GO) Brazil \\ A. T. Avelar, \\ Instituto de Física, Universidade de Brasília, 70.919-970, Brasília, DF, Brazil and \\ Instituto de Física, Universidade Federal de Goiás, 74.001-970, Goiânia, GO Brazil \\ and J. M. C. Malbouisson* \\ Department of Physics, University of Alberta, Edmonton, AB T6G 2J1, Canada \\ Received on 29 October, 2005
}

\begin{abstract}
We study two experimental schemes generating highly excited number states starting from an initial squeezed state. One of the schemes works for stationary fields, the other for traveling ones.
\end{abstract}

\section{INTRODUCTION}

Considerable theoretical and experimental efforts have been devoted to the preparation and characterization of nonclassical states of the electromagnetic field, such as subPoissonian [1], squeezed [2], entangled [3] and "Schrödingercat" states [4]. According to a theorem by Hillery [5], all pure states which are not coherent are non classical and, according to a criterion by Lee [6], the number states exhibit maximum nonclassical depth, hence standing as the most non classical states. Now, although they play the role of a "battle-horse" in the formalism of quantum theory, their preparation in laboratories is difficult: in spite of various proposals for their generation [7], till now only number states having small numbers $(N=1,2)$ have been prepared in laboratories [8]. Recently, an elegant proposal for generation of large number states $(N \sim 6)$ was presented [9], the scheme standing for fields trapped inside a high-Q cavity-QED. Other important works concerning this topic are found in [10].

Many of the earlier proposals cited in [7] employ superpositions of circular coherent states for generating number states. Recently, we have shown that highly excited Fock states of the kind $\left|2^{N}\right\rangle$ (and some of their simple superpositions) can be obtained from specific superpositions of circular coherent states $[11,12]$. In the present work, we consider superpositions of circular squeezed states (SCSS) which lead to the generation of a broader class of number states. We show that such states may result in a number state, of the type $\left|k 2^{N}\right\rangle$, with $k=1,2,3, \ldots$ and $N=2,3,4, \ldots$, for convenient choices of the involved parameters. We will consider the construction of these states in two distinct schemes, one of them being concerned with states of stationary fields inside a microwave cavity, in which $N$ stands for the number of atoms rotating the squeezed states in phase space when those atoms cross the cavity; the other scheme refers to traveling fields, where $N$ stands for the number of apparatus rotating the circular squeezed states. Both schemes are inspired on others exist-

*Permanent adress: Instituto de Física, Universidade Federal da Bahia, 40.210-310, Salvador, BA, Brazil ing in the literature, used for other proposals.

This paper is arranged as follows. In Section II we study the preparation of the SCSS for stationary fields and in Section III we do the same for traveling fields. As we will see below the SCSS furnishes our wanted family of number states as a by-product. In the Section IV we show how to obtain highly excited number states with good fidelity, of the family $\left|k 2^{N}\right\rangle$, by sculpturing a convenient initial state [13]. The Section V contains the comments and conclusion.

\section{PRODUCTION OF SCSS FOR STATIONARY FIELDS}

To produce a SCSS as a stationary field inside a microwave cavity we start from a single-mode squeezed state initially prepared in it. (A recent proposal preparing such an initial state was presented in [14].) The present scheme relies on selective atomic detection of an entangled state-vector describing the whole atom-field system. The procedure is inspired on others appearing previously in the literature $[15,16]$. The method employs a high-Q superconducting cavity $\mathrm{C}$, placed between two low-Q cavities (Ramsey zones $\mathrm{R}_{1}$ and $\mathrm{R}_{2}$ ), as schematically shown in Fig. 1.

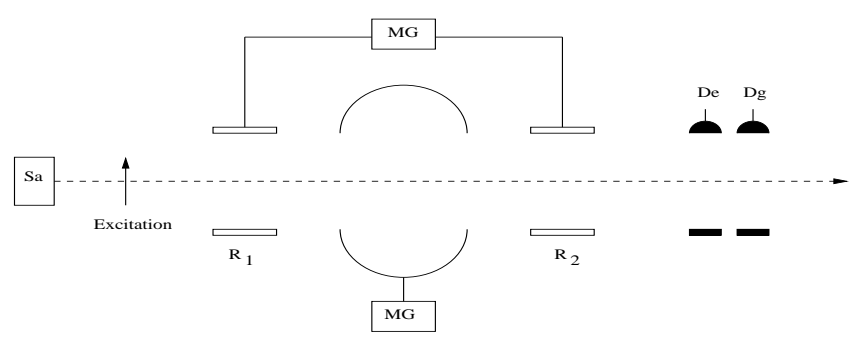

FIG. 1: Schematical illustration of the experimental setup for production of SCSS in a high-Q cavity using dispersive atomic probes. $\mathrm{S}_{A}$ and MG stand for "source of atoms" and "microwave generator", respectively; $\mathrm{D}_{e}\left(\mathrm{D}_{g}\right)$ is a selective detector for state $|e\rangle(|g\rangle)$.

Highly excited (Rydberg) atoms are prepared in circular state $|e\rangle$ (principal quantum number $n=51$ in the case of rubidium) via an appropriate laser beam. These atoms are sent, one by one, throughout the system as shown in Fig.1. In the 
Ramsey zones, classical fields produced by a MG interact resonantly with the transition between $|e\rangle$ and $|g\rangle(n=50)$ leading to the atomic state $|e\rangle \rightarrow|e\rangle+|g\rangle$ and $|g\rangle \rightarrow|g\rangle-|e\rangle$. A third (auxiliary) atomic level $|i\rangle(\mathrm{n}=52)$ is crucial in the scheme [17]: the cavity frequency is adjusted close to resonance (detuned by small $\delta$ ) with the transition $|e\rangle \longrightarrow|i\rangle$, but far from the transition $|g\rangle \longrightarrow|e\rangle$. The number of photons in the field being denied to change, only the phase may vary. So, concerning with the transition $|g\rangle \longleftrightarrow|e\rangle$ the atom-field interaction in $\mathrm{C}$ is dispersive, instead of resonant. Such an interaction is described by the effective atom-field Hamiltonian $[17,18]$

$$
\hat{H}_{\text {int }}=\hbar \omega_{\mathrm{eff}} \hat{a}^{\dagger} \hat{a}(|i\rangle\langle i|-| e\rangle\langle e|),
$$

with $\omega_{\text {eff }}=2 d^{2} / \delta, d$ being the atomic dipole moment. Thus the atom crossing the cavity produces a phase-shift in the field state when it is in the excited state $|e\rangle$, but no phase-shift occurs when the atom is in the ground state $|g\rangle$.

Consider that the field in the high-Q cavity is initially in a squeezed state $|\alpha, z\rangle=\hat{D}(\alpha) \hat{S}(z)|0\rangle$, where $\hat{D}(\alpha)=e^{\alpha \hat{a}^{\dagger}-\alpha^{*} \hat{a}}$ and $\hat{S}(z)=e^{\frac{1}{2} z^{*} \hat{a}^{2}-\frac{1}{2} z \hat{a}^{\dagger 2}}$ are the (unitary) displacement and squeeze operators, respectively, with $\alpha$ and $z$ standing for the corresponding displacement and squeeze parameters [19]. The evolution of the entangled atom-field state, as the first atom crosses the system, follows the steps presented in Table 1 below (up to normalizations and using $e^{i \phi \hat{a}^{\dagger}} \hat{a}|\alpha, z\rangle=$ $\left.\left|e^{i \phi} \alpha, e^{2 i \phi} z\right\rangle[20]\right)$.

TABLE 1. Evolution of the atom-field state during the passage of the first atom throughout the system.

\begin{tabular}{cc}
\hline \hline $1^{\text {st }}$ atom & $|\Psi\rangle_{\text {atom+field }}$ \\
\hline before $\mathrm{R}_{1}:$ & $|e\rangle_{1}|\alpha, z\rangle$ \\
after $\mathrm{R}_{1}:$ & $\left(|g\rangle_{1}+|e\rangle_{1}\right)|\alpha, z\rangle$ \\
after $\mathrm{C}:$ & $|g\rangle_{1}|\alpha, z\rangle+|e\rangle_{1}\left|e^{i \theta_{1}} \alpha, e^{2 i \theta_{1}} z\right\rangle$ \\
after $\mathrm{R}_{2}:$ & $|g\rangle_{1}\left(\left|e^{i \theta_{1}} \alpha, e^{2 i \theta_{1}} z\right\rangle+|\alpha, z\rangle\right)+|e\rangle_{1}\left(\left|e^{i \theta_{1}} \alpha, e^{2 i \theta_{1}} z\right\rangle-|\alpha, z\rangle\right)$ \\
\hline \hline
\end{tabular}

In table 1 subscript 1 refers to the first atom and $\theta_{1}=\omega_{\mathrm{eff}} t_{1}$, $t_{1}$ being the time the atom takes to cross the cavity. Now, if this first atom is detected in the state $|g\rangle(|e\rangle)$, the field in the cavity is projected onto the state $\left|e^{i \theta_{1}} \alpha, e^{2 i \theta_{1}} z\right\rangle+|\alpha, z\rangle$ $\left(\left|e^{i \theta_{1}} \alpha, e^{2 i \theta_{1}} z\right\rangle-|\alpha, z\rangle\right)$. For the present purpose we take the detection being that of the ground state $|g\rangle$.

If a second atom is ejected after the detection of the first one in $|g\rangle$ (the field state in the cavity being $\left|e^{i \theta_{1}} \alpha, e^{2 i \theta_{1}} z\right\rangle+$ $|\alpha, z\rangle)$, the whole atom-field state now evolves as shown in Table 2.

TABLE 2. Same as in Table 1, for the second atom.

\begin{tabular}{cc}
\hline \hline $2^{\text {nd }}$ atom & $|\Psi\rangle_{\text {atom }+ \text { field }}$ \\
\hline before $\mathrm{R}_{1}:$ & $|e\rangle_{2}\left(\left|e^{i \theta_{1}} \alpha, e^{2 i \theta_{1}} z\right\rangle+|\alpha, z\rangle\right)$ \\
after $\mathrm{R}_{1}:$ & $\left(|g\rangle_{2}+|e\rangle_{2}\right)\left(\left|e^{i \theta_{1}} \alpha, e^{2 i \theta_{1}} z\right\rangle+|\alpha, z\rangle\right)$ \\
after $\mathrm{C}:$ & $|g\rangle_{2}\left(\left|e^{i \theta_{1}} \alpha, e^{2 i \theta_{1}} z\right\rangle+|\alpha, z\rangle\right)+|e\rangle_{2}\left(\left|e^{i\left(\theta_{1}+\theta_{2}\right)} \alpha, e^{2 i\left(\theta_{1}+\theta_{2}\right)} z\right\rangle+\left|e^{i \theta_{2}} \alpha, e^{2 i \theta_{2}} z\right\rangle\right)$ \\
after $\mathrm{R}_{2}:$ & $|g\rangle_{2}\left(\left|e^{i \theta_{1}} \alpha, e^{2 i \theta_{1}} z\right\rangle+|\alpha, z\rangle+\left|e^{i\left(\theta_{1}+\theta_{2}\right)} \alpha, e^{2 i\left(\theta_{1}+\theta_{2}\right)} z\right\rangle+\left|e^{i \theta_{2}} \alpha, e^{2 i \theta_{2}} z\right\rangle\right)+|e\rangle_{2}\left(-\left|e^{i \theta_{1}} \alpha, e^{2 i \theta_{1}} z\right\rangle\right.$ \\
& $\left.|-| \alpha, z\rangle+\left|e^{i\left(\theta_{1}+\theta_{2}\right)} \alpha, e^{2 i\left(\theta_{1}+\theta_{2}\right)} z\right\rangle+\left|e^{i \theta_{2}} \alpha, e^{2 i \theta_{2}} z\right\rangle\right)$ \\
\hline
\end{tabular}

We see that detecting the second atom again in $|g\rangle$ leads to a superposition of the state $\left|e^{i \theta_{1}} \alpha, e^{2 i \theta_{1}} z\right\rangle+|\alpha, z\rangle$ with its rotated (by $\theta_{2}$ ) partner $\left|e^{i\left(\theta_{1}+\theta_{2}\right)} \alpha, e^{2 i\left(\theta_{1}+\theta_{2}\right)} z\right\rangle+\left|e^{i \theta_{2}} \alpha, e^{2 i \theta_{2}} z\right\rangle$. Pursing further in this way, after the passage of $N$ atoms throughout the system, controlling their velocities such that $\theta_{1}=\theta$, $\theta_{2}=\theta / 2, \ldots, \theta_{N}=\theta / 2^{N-1}$ and all atoms being detected in the state $|g\rangle$, the state generated in the cavity results

$$
\left|\Psi_{N}(\alpha, z, \theta)\right\rangle=\mathcal{N}_{N} \sum_{j=0}^{J_{N}}\left(\left|e^{i \theta} \alpha_{j}, e^{2 i \theta} z_{j}\right\rangle+\left|\alpha_{j}, z_{j}\right\rangle\right),
$$

where $\alpha_{j}=\alpha \exp \left(i \theta j / 2^{N-1}\right), z_{j}=z \exp \left(i \theta j / 2^{N-2}\right)$ and $J_{N}=$ $2^{N-1}-1$. In the foregoing procedure the final field-state pro- 
duced by detection of the $(j-1)$-th atom stands for the initial normalization factor $\mathcal{N}_{N}$ is given by field-state in the cavity when the $j$-th atom is released. The

$$
\left|\mathcal{N}_{N}(\alpha, z, \theta)\right|^{-2}=\exp \left[-\alpha^{2} e^{z} \operatorname{sech}(z)\right] \operatorname{sech}(z) \mathcal{A}_{N}(\alpha, z, \theta),
$$

where

$$
\begin{aligned}
\mathcal{A}_{N}(\alpha, z, \theta)= & \sum_{j, j^{\prime}=0}^{J_{N}}\left\{\frac{2}{\sqrt{1-\tanh ^{2}(z) \exp \left(2 i \Theta_{j j^{\prime}}\right)}} \exp \left[\frac{\alpha^{2} e^{2 z} \operatorname{sech}^{2}(z) \exp \left(i \Theta_{j j^{\prime}}\right)}{1+\tanh (z) \exp \left(i \Theta_{j j^{\prime}}\right)}\right]\right. \\
& +\frac{1}{\sqrt{1-\tanh ^{2}(z) \exp \left[2 i\left(\Theta_{j j^{\prime}}+\theta\right)\right]}} \exp \left[\frac{\alpha^{2} e^{2 z} \operatorname{sech}^{2}(z) \exp \left[i\left(\Theta_{j j^{\prime}}+\theta\right)\right]}{1+\tanh (z) \exp \left[i\left(\Theta_{j j^{\prime}}+\theta\right)\right]}\right] \\
& \left.+\frac{1}{\sqrt{1-\tanh ^{2}(z) \exp \left[2 i\left(\Theta_{j j^{\prime}}-\theta\right)\right]}} \exp \left[\frac{\alpha^{2} e^{2 z} \operatorname{sech}^{2}(z) \exp \left[i\left(\Theta_{j j^{\prime}}-\theta\right)\right]}{1+\tanh (z) \exp \left[i\left(\Theta_{j j^{\prime}}-\theta\right)\right]}\right]\right\},
\end{aligned}
$$

with $\Theta_{j j^{\prime}}=\theta\left(j-j^{\prime}\right) / 2^{N-1}$.

The squeezed states participating of the superposed state (2) can be represented by equally separated ellipses over a circle of radius $|\alpha|$ in the complex plane, ranging in the interval $\left[\theta_{0}, \theta_{0}+2 \theta\left(1-2^{-N}\right)\right]$ where $\theta_{0}=\arg (\alpha)$, as illustrated in Fig. 2 taking $\theta_{0}=0$ and $\theta=\pi$. Without loss of generality we take $\alpha$ and $z$ to be real and positive from now on. Finally, the state $\left|\Psi_{N}(\alpha, \theta, z)\right\rangle$ given in Eq.(2) constitutes our (auxiliary) SCSS.

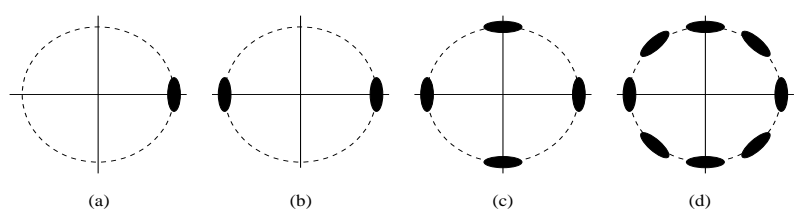

FIG. 2: Pictorial representation of $3^{\text {rd }}$-order sequence leading to $\left|\Psi_{N}\right\rangle$, with $N=3$, in the phase-space: (a) $\left|\Psi_{0}\right\rangle$, (b) $\left|\Psi_{1}\right\rangle$, (c) $\left|\Psi_{2}\right\rangle$ and (d) $\left|\Psi_{3}\right\rangle ;\left|\Psi_{0}\right\rangle=|\alpha, z\rangle=D(\alpha) S(z)|0\rangle$.

\section{PRODUCTION OF SCSS FOR TRAVELING FIELDS}

The superposition state (2) can also be produced as running waves of the electromagnetic field. In this sense the generation scheme is complementary to the previous one, while constituting a mapping, step by step, of one onto another. It consists of a set of Mach-Zehnder interferometers (MZI), all them fed with a Fock state $|1\rangle$ and the vacuum in the internal modes ( $b$ and $c$ respectively). Each of the MZI contains a Kerr-medium in one of the arms that couples the internal $b$-mode to an external mode $(a)$, which is initially prepared in a squeezed state $|\alpha, z\rangle$ [21]. The experimental setup is illustrated in Fig. 3: (A) shows details of the first MZI including a Kerr-media, a device proposed to generate optical Schrödinger cat states [22], which has recently proved to permit the generation of superpositions of two squeezed states in running modes [23]; in (B) one presents pictorially the se- quence of MZI which can also be used to to generate superpositions of circular coherent states $[11,12]$.
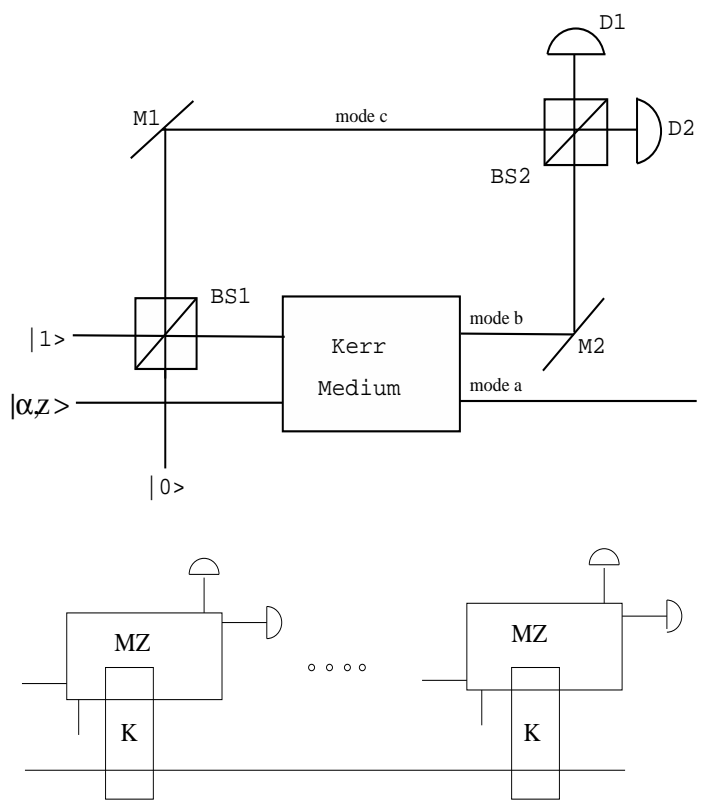

FIG. 3: Schematical illustration of the setup producing the SCSS as running modes; (A) shows details of the first MZI including the nonlinear Kerr-medium, while (B) depicts the sequence of MZI plus associated Kerr-media.

Initially a single photon (mode b) and vacuum state (mode c) enter in the first ideal 50/50 symmetric beam splitter (BS) of the MZI. The action of BS1 is described, in terms of the annihilation operators $\hat{b}$ and $\hat{c}$ for modes $\mathrm{b}$ and $\mathrm{c}$, by the unitary operator [24]

$$
\hat{R}_{b c}=\exp \left[i \frac{\pi}{4}\left(\hat{b}^{\dagger} \hat{c}+\hat{b} \hat{c}^{\dagger}\right)\right] .
$$

Just after the BS1 the (unnormalized) state of the system is given by $\left(|1\rangle_{b}|0\rangle_{c}+i|0\rangle_{b}|1\rangle_{c}\right)|\alpha, z\rangle_{a}$, where $|\alpha, z\rangle_{a}$ is the initial squeezed state, previously prepared in a running wave [21] 
in the mode $a$. The dispersive Kerr interaction between $a$ - and $b$-modes is described by the interaction Hamiltonian [25]

$$
\hat{H}_{K}=\hbar K \hat{a}^{\dagger} \hat{a} \hat{b}^{\dagger} \hat{b}
$$

where $K$ is proportional to the third order nonlinear susceptibility of the medium. Therefore the action of the Kerr-medium is represented by the unitary operator

$$
\hat{U}_{K}=\exp \left(-i \theta \hat{a}^{\dagger} \hat{a} \hat{b}^{\dagger} \hat{b}\right)
$$

where $\theta=K l / v, l$ being the length of the Kerr-medium and $v$ the velocity of light in the medium. So, after the medium and just before the second beam splitter BS2, the (unnormalized) state of the system reads $|1\rangle_{b}|0\rangle_{c}\left|\alpha e^{-i \theta}, z e^{-i 2 \theta}\right\rangle_{a}+$ $i|0\rangle_{b}|1\rangle_{c}|\alpha, z\rangle_{a}$. The second beam splitter implements the transformation

$$
\begin{aligned}
|1\rangle_{b}|0\rangle_{c} & \rightarrow\left(|1\rangle_{b}|0\rangle_{c}+i|0\rangle_{b}|1\rangle_{c}\right) / \sqrt{2} \\
|0\rangle_{b}|1\rangle_{c} & \rightarrow\left(|0\rangle_{b}|1\rangle_{c}+i|1\rangle_{b}|0\rangle_{c}\right) / \sqrt{2}
\end{aligned}
$$

Thus, after BS2 the entangled state of the system is

$$
\begin{aligned}
|\Psi\rangle_{A F}= & \frac{1}{2}\left[|1\rangle_{b}|0\rangle_{c}\left(\left|e^{-i \theta} \alpha, e^{-2 i \theta} z\right\rangle_{a}-|\alpha, z\rangle_{a}\right)\right. \\
& \left.+i|0\rangle_{b}|1\rangle_{c}\left(\left|e^{-i \theta} \alpha, e^{-2 i \theta} z\right\rangle_{a}+|\alpha, z\rangle_{a}\right)\right] .
\end{aligned}
$$

If detector D1 (D2) fires signaling the detection of the state $|1\rangle_{b}|0\rangle_{c}\left(|0\rangle_{b}|1\rangle_{c}\right)$, the a-mode is projected onto the state $\left|e^{-i \theta} \alpha, e^{-2 i \theta} z\right\rangle_{a}-|\alpha, z\rangle_{a}\left(\left|e^{-i \theta} \alpha, e^{-2 i \theta} z\right\rangle_{a}+|\alpha, z\rangle_{a}\right)$; so, taking the internal photon detected in D2 (mode $c)$, the state projected in mode $a$ is $\left|\Psi_{1}(\alpha,-\theta, z)\right\rangle$ [22].
Next, assume that a second MZI is aligned with the first one, again with a single photon in mode $b$ and the vacuum in mode $c$, but with mode $a$ in the state $\left|e^{-i \theta} \alpha, e^{-2 i \theta} z\right\rangle_{a}+|\alpha, z\rangle_{a}$, the outcome of the first MZI. If the second Kerr-medium is adjusted such that $\theta=\theta_{2}$, then the field-field entangled state in the second apparatus evolves as in Table 2 with $|e\rangle_{2}\left(|g\rangle_{2}\right)$ replaced by $|1\rangle_{b}|0\rangle_{c}\left(i|0\rangle_{b}|1\rangle_{c}\right)$ and $\theta_{1}\left(\theta_{2}\right)$ being changed to $-\theta_{1}\left(-\theta_{2}\right)$. Going further ahead, we consider a sequence of $N$ MZI [26]; taking $\theta_{j}=\theta / 2^{j-1}$ and assuming that all photons are detected by D2 (corresponding to the detection of the state $|0\rangle_{b}|1\rangle_{c}$ ), the outcome in mode $a$ will be the state $\left|\Psi_{N}(\alpha,-\theta, z)\right\rangle$. Notice that $\left|\Psi_{N}(\alpha, \theta+2 \pi, z)\right\rangle=\left|\Psi_{N}(\alpha, \theta, z)\right\rangle$ so that these states coincide with the symmetrical superposition for the cases $\theta= \pm \pi$.

The schemes for generating SCSS for trapped fields inside a cavity and for running fields can be mapped one onto another as alluded before. The beam-splitters in the second scheme play the role of the Ramsey zones in the first one whereas the atom-field interaction in the first scheme is translated to the field-field interaction engendered by the Kerr-medium used in the second one. Furthermore, the ionization detection of the atomic state $|g\rangle$ is equivalent to the photon register in the detector D2 and, naturally, the number of atoms crossing the cavity-system corresponds to the number of aligned MZ apparatus of the second scheme, since both correspond to the recursive procedure rotating squeezed states around a circle in the phase space.

\section{GETTING NUMBER STATES FROM THE SCSS}

The photon-number distribution (PND) of the SCSS, $P_{N}(n ; \alpha, z, \theta)=\left|\left\langle n \mid \Psi_{N}(\alpha, z, \theta)\right\rangle\right|^{2}$, is given by

$$
P_{N}(n ; \alpha, z, \theta)=\frac{1}{\mathcal{A}_{N}(\alpha, z, \theta)} \frac{(\tanh z)^{n}}{2^{n} n !} H_{n}^{2}\left(\frac{\alpha e^{z}}{\sqrt{\sinh (2 z)}}\right) \frac{2 \sin ^{2}(n \theta)}{1-\cos \left(n \theta / 2^{N-1}\right)}
$$

where $H_{n}(x)$ are the Hermite polynomials. The last factor in the above expression (which carries the $\theta$-dependence of $\left.P_{N}(n ; \alpha, z, \theta)\right)$ vanishes for many values of $n$ whenever $\theta$ is a rational fraction of $\pi$. This feature is maximized if one takes $\theta=\pi$ and, in this case, the only occupied number states are those for which $n$ is a multiple of $2^{N}$. Actually, in the limit $\theta \rightarrow \pi$, the last factor in (11) reduces to $2^{2 N} \delta_{n, k 2^{N}}$, where $k=0,1,2, \ldots$; in other words, $\left|\Psi_{N}(\alpha, z, \pi)\right\rangle$ is a superposition containing solely number states of the kind $\left|k 2^{N}\right\rangle$. As it is shown below, depending on the appropriated choices of the parameters $\alpha$ and $z$, such state becomes a single number state $\left|k 2^{N}\right\rangle$ with a very high fidelity.

To investigate whether a SCSS corresponds to a number state of the type $\left|k 2^{N}\right\rangle$ one should analyze the fidelity between the states $\left|k 2^{N}\right\rangle$ and $\left|\Psi_{N}(\alpha, z, \pi)\right\rangle$, which we denote by $\mathcal{F}$. This quantity is obtained from Eq. (11) by taking $\theta=\pi$ and $n=k 2^{N}$ :

$$
\mathcal{F}\left(\left|\Psi_{N}(\alpha, z, \pi)\right\rangle,\left|k 2^{N}\right\rangle\right)=\frac{2^{2 N}}{\mathcal{A}_{N}(\alpha, z, \pi)} \frac{(\tanh z)^{k 2^{N}}}{2^{k 2^{N}}\left(k 2^{N}\right) !} H_{k 2^{N}}^{2}\left(\frac{\alpha e^{z}}{\sqrt{\sinh (2 z)}}\right)
$$

As a general procedure, for fixed values of $N$ and $k$, one should search for the values of $\alpha$ and $z$ which maximize the fidelity
(12); as closer the maximum value of this fidelity is to 1 , as 
better $\left|\Psi_{N}(\alpha, z, \pi)\right\rangle$ approximates the number state $\left|k 2^{N}\right\rangle$. The behavior of $\mathcal{F}$ as a function of $\alpha$ and $z$ is presented in Fig.4 for a specific choice of $N$ and $k$.

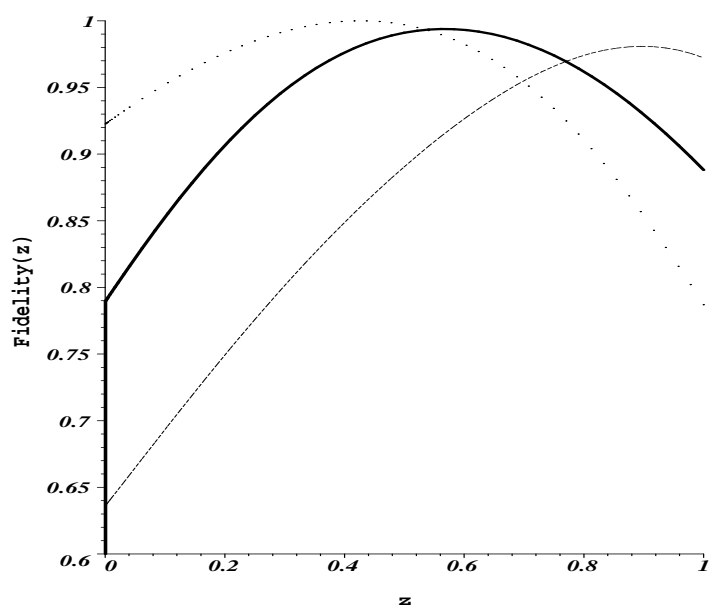

FIG. 4: Fidelity between the state $|\Psi(\alpha, z, \pi)\rangle$ and the states $\left|k 2^{N}\right\rangle$, as function $z$, for some values of $\alpha$ : 3.0 (dotted line); 4.0 (full line), 5.0 (dashed line).

A simple way to illustrate the generation of the desired number states of the type $\left|k 2^{N}\right\rangle$ consists in plotting the PND of the state $\left|\Psi_{N}(\alpha, z, \pi)\right\rangle$, after choosing convenient values of the parameters $\alpha$ and $z$, and making $N$ run from 1 to $2,3,4, \ldots$; this is presented in Figs.5-7 for some cases. The process works as if the atoms sculpture the initial field state $\left|\Psi_{0}\right\rangle=|\alpha, z\rangle$, leading it to a final number state. The name sculpture relies on the action of atoms removing components of the initial state until achieving the final state, as we see in Figs.5-7.

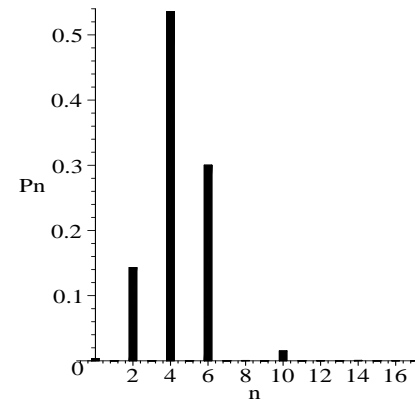

(a) $N=1$

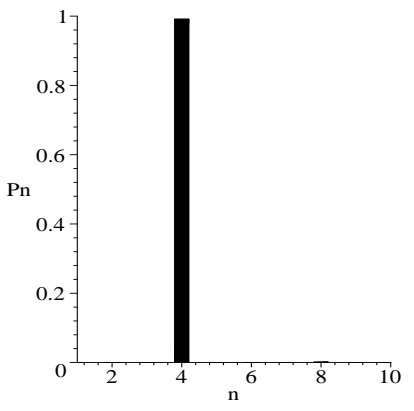

(b) $N=2$
FIG. 5: Plots of PND of SCSS as function of $\mathrm{n}$ for $\alpha=2.0, z=0.6$ showing the sculpture of the initial state $|\alpha, z\rangle$ yielding the number state $|4\rangle$; it coincides with $\left|k 2^{N}\right\rangle$ for $k=1, N=2$.

Figure 5 corresponds to the member $|4\rangle$ of the family of number states $\left|k 2^{N}\right\rangle$ for $k=1, N=2$. Fig. 6 stands for the number state $|24\rangle$, for $k=3, N=3$ and Fig. 7 is for the number state $|48\rangle$, for $k=3, N=4$.

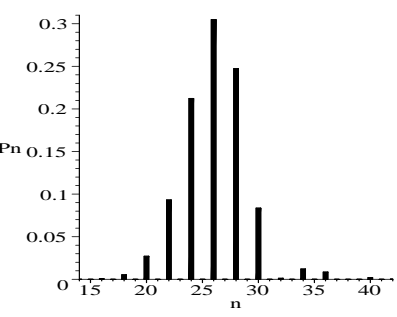

(a) $N=1$

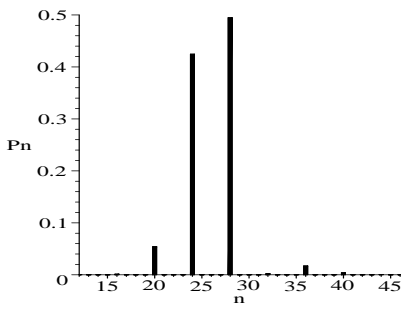

(b) $N=2$

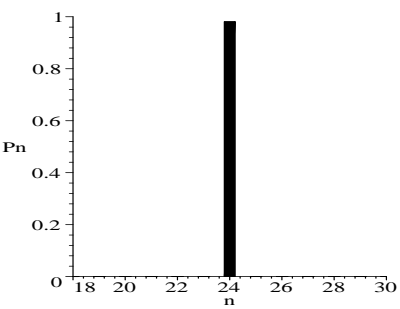

(c) $N=3$

FIG. 6: Same as Fig.(4) for $\alpha=5.0$ and $z=0.9$, yielding the number state $|24\rangle$; it coincides with $\left|k 2^{N}\right\rangle$ for $k=3, N=3$.

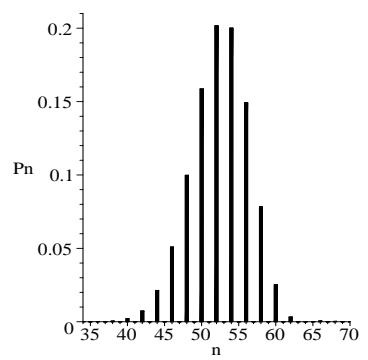

(a) $N=1$

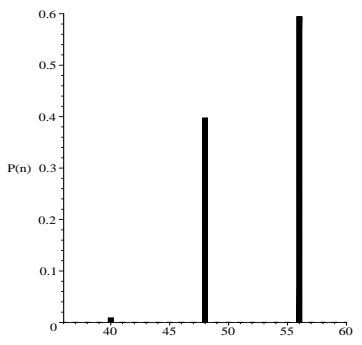

(c) $N=3$

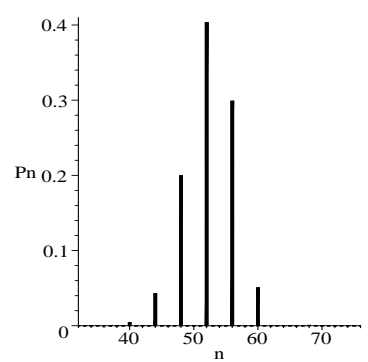

(b) $N=2$

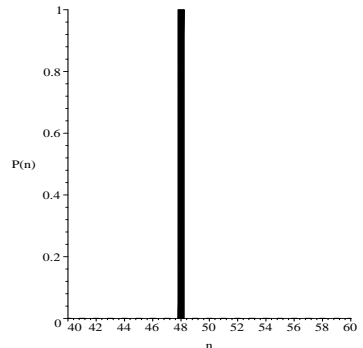

(d) $N=4$
FIG. 7: Same as Fig.(4), for $\alpha=7.2, z=0.7$, leading to the state $|48\rangle$; it coincides with $\left|k 2^{N}\right\rangle$ for $k=3, N=4$. 


\section{COMMENTS AND CONCLUSION}

We have presented proposals preparing a single-mode of the electromagnetic field in highly excited number states of the type $\left|k 2^{N}\right\rangle$. One of these schemes stands for stationary waves (Fig.1), the other standing for traveling waves (Fig.3). The strategy has consisted in preparing the SCSS for each case, the number states of the family $\left|k 2^{N}\right\rangle$ emerging from the SCSS by firstly choosing convenient values of the parameters $\alpha, z$ characterizing the initial squeezed state $\left|\Psi_{0}\right\rangle=|\alpha, z\rangle$ and next rotating it $N$ times around a circle in the phase space. For stationary fields $N$ refers to number of atoms crossing the microwave cavity; for traveling fields $N$ refers to the number of MZI (plus Kerr-medium) composing the whole apparatus. As result, the sculpture using 2 atoms furnishes only the state $|4\rangle$, with fidelity $\mathcal{F}=99.1 \%$ and success probability $\mathcal{P}_{N}=1 / 2^{N}=1 / 4$ for $N=2$ (Fig.5). However, sculpture using 3 atoms (Fig.6) furnishes various number states (not shown in figures, exception to $|24\rangle$ ), such as: $|8\rangle$, for $\alpha=3.0$ and $z=0.4$, with $\mathcal{F}=99.9 \% ;|16\rangle$, for $\alpha=4.0$ and $z=0.6$, with $\mathcal{F}=99.3 \% ;|24\rangle$, with $\mathcal{F}=98.0 \%$; and also the states $|32\rangle$ and $|40\rangle$, both having fidelity near $97 \%$. All these number states have same success probability, $\mathcal{P}_{3}=1 / 2^{3}=1 / 8$. When the sculpture uses 4 atoms we obtain the number states: $|16\rangle$, $|32\rangle,|48\rangle$ (see Fig.7), $|64\rangle,|80\rangle,|96\rangle$ and $|112\rangle$, all having fidelity greater than $99.9 \%$ and same success probability: $\mathcal{P}_{4}=$ $1 / 2^{4}=1 / 16$.

It is worth emphasizing that highly excited Fock states, for stationary and traveling fields, can also be obtained in a second scenario, starting from an initial coherent state [11]. From a practical view point this is advantageous since preparation of squeezed states requires one step beyond the preparation of coherent states. From a theoretical view point the case of coherent state is a particularization of the present approach by setting $z=0$ in the initial state describing the atom-field system, namely: $|\Psi\rangle_{A F}=|e\rangle|\alpha, z\rangle$ going to $|\Psi\rangle_{A F}=|e\rangle|\alpha\rangle$. However, in spite of additional technological difficulties, the results obtained for squeezed states are better than those found for coherent states. For example, when two atoms cross the cavity one finds $|n\rangle_{S S}=|4\rangle$ while no number state $|n\rangle_{C S}$ is found [subscripts $\mathrm{SS}(\mathrm{CS})$ stands for squeezed(coherent) state]. Also, for $\mathrm{N}$ atoms crossing the cavity, using an initial SS furnishes the family of number states $|n\rangle_{S S}=\left|k 2^{N}\right\rangle$ while using an initial CS only furnishes the number state $|n\rangle_{C S}=\left|2^{N}\right\rangle$. Hence, for each (convenient) value of $\alpha$ and a number of atoms, one obtains $N_{S S}$ greater than $N_{C S}$.

Here we have assumed all atomic and field detectors as ideal. Recent technological advances yield photodetector efficiency near $100 \%$ [27]; till now, atomic detectors are not so good as photodetectors, the best efficiency being near $70 \%$ [28]. The use of non-ideal detectors usually causes deleterious influence, reducing the fidelity of the state being prepared. On the other hand both schemes depend on experimental im- provements: highly excited stationary fields in number states $|n\rangle$, inside a cavity, are strongly affected by decoherence effects degrading the state being prepared. Accordingly [29], the decoherence time is $\tau_{d} \simeq \tau_{c a v} / N, \tau_{c a v}$ being the lifetime of the field created inside the cavity, showing $\tau_{d}$ diminishing when $N$ grows. For traveling fields the decoherence effect is not so crucial.

Concerning with the reliability of our experimental scheme for the stationary case, we first mention that a $\pi$ phase-shift in the field - induced by the passage of the first atom throughout the apparatus (Fig. 1) - has been achieved in [4] for creating Schrödinger cat states. The present scheme, however, needs a sequence of phase-shifts: $\pi, \pi / 2, \pi / 4, \ldots, \pi / 2^{N}$, which requires adequate velocity selectors to choose appropriated speeds for atoms crossing the cavity. In addition, improved time switches should be coupled with fine microwave generators that properly adjust the intensities of the Ramsey zones to produce $\pi / 2$ pulse in the atomic states. In this way one prepares the sequence of atoms engendering the desired phase-shifts in all steps of the process. But one has also to warrant that all Rydberg atoms are detected in their ground states. Roughly speaking, the probability that $N$ atoms are all detected in $|g\rangle$ is $2^{-N}$ and one has to ensure that the time separation between the passage of two consecutive atoms is shorter than the decoherence time of the field already generated in the cavity. Therefore, subtle experimental aspects are involved in the production of highly excited Fock states in a cavity, but in principle the procedure can be implemented, at least for moderate number of atoms.

When concerning with running waves, some distinct aspects should be considered. The first point is the possibility of generating a phase-shift of $\pi$ with the first MZ. For optical modes, to obtain such a phase-shift would require a very large Kerr nonlinear susceptibility or an extremely long medium [30]. This point is a great difficulty for the generation of Fock states of the type $\left|k 2^{N}\right\rangle$ in running waves, although some suggestions to enhance the Kerr nonlinearity have been presented [31]. Besides this aspect one has to produce synchronized single photon states to feed modes $b$ of all MZI, align them and adjust Kerr media to guarantee that phase-shift produced by the $\mathrm{j}$-th MZI is given by $\pi / 2^{j-1}$. Solution to this difficulty has been addressed recently [32]. Even under such restrictive conditions, some states of the type $\left|k 2^{N}\right\rangle$ might be created as travelling waves for moderate number of MZI. We hope that remaining difficulties could be circumvented in near future by technological advances.

\section{Acknowledgments}

We thank the CNPq, Brazilian Agency, for the partial support. 
al., Phys. Rev. Lett. 86, 3534 (2001).

[2] R. E. Slusher et al., Phys. Rev. Lett. 55, 2409 (1985); L. A. Wu et al., Phys. Rev. Lett. 57, 2520 (1986); R. E. Slusher et al., J. Opt. Soc. Am. B 4, 1453 (1987).

[3] C.H. Bennett, S.J. Wiesner, Phys. Rev. Lett. 69, 2881 (1992); C.H. Bennett et al., Phys. Rev. Lett. 70, 1895 (1993); C.H. Bennett, D.P. Di Vincenzo, Nature, 404, 247 (2000).

[4] See, e.g., L. Davidovich, M. Brune, J. M. Raimond, and S. Haroche, Phys. Rev. A 53, 1295 (1996); M. Brune et al., Phys. Rev. Lett. 77, 4887 (1996).

[5] M. Hillery, Phys. Rev. A 35, 725 (1987).

[6] C. T. Lee, Phys. Rev. A 44, R2775 (1991); Phys. Rev. A 45 (1992) 6586; Phys. Rev. A 52, 3374 (1995).

[7] L. Mandel, Opt. Lett. 4, 205 (1979); Phys. Rev. Lett. 49, 136 (1982); P. Filipowicz, J. Javanainen, and P. Meystre, J. Opt. Soc. Am. B 3, 906 (1986); J. Krause, M. O. Scully, and H. Walther, Phys. Rev. A 36, 4547 (1987); J. Krause et al., Phys. Rev. A 39, 1915 (1989); J. J. Slosser, P. Meystre, and S. L. Braunstein, Phys. Rev. Lett. 63, 934 (1989); M. J. Holland, D. F. Walls, and P. Zoller, Phys. Rev. Lett. 67, 1716 (1991); G. S. Agarwal et al., Phys. Rev. A 49, 4077 (1994); P. Domokos, J. Janszky, and P. Adam, Phys. Rev. A50, 3340 (1994); J. Janszky, P. Domokos, S. Szabó, and P. Adam, Phys. Rev. A 51, 4191 (1995); S. Y. Kilin and D. B. Horoshko, Phys. Rev. Lett. 74, 5206 (1995); S. Szabo et al., Phys. Rev. A 53, 2698 (1996); G. Harel, G. Kurizki, Phys. Rev. A 54, 5410 (1996); W. Leonski, Phys. Rev. A 54, 3369 (1996); R. L. de Matos Filho, W. Vogel, Phys. Rev. Lett. 76, 608 (1996); O. Steuernagel, Opt. Commun. 138, 71 (1997); H. Saito, M. Ueda, Phys. Rev. Lett. 79, 3869 (1997); P. Domokos et al., Eur. Phys. J. D 1, 1 (1998); W.D. Jose, S.S. Mizrahi, J. Opt. B: Quantum Semiclass Opt. 2, 306 (2000).

[8] For preparation of 1-photon state inside a cavity, see: S. Brattke, BT.H. Varcoe, H. Walther, Phys. Rev. Lett. 86, 3534 (2001); and refs. therein. For 2-photon state, see: B.T.H. Varcoe et al., Nature 403, 743 (2000); P. Bertet et al., Phys. Rev. Lett. 88, 143601 (2002).

[9] M. F. Santos, E. Solano, and R.L. de Matos Filho, Phys. Rev. Lett. 87, 93601 (2001).

[10] P. Michler et al., Nature 406, 968 (2000); S. Brattke et al., Phys. Rev. Lett. 86, 3534 (2001).
[11] L. P. A. Maia et al., J. Opt. B: Quantum Semiclass. Opt. 6, 351 (2004).

[12] A. Aragão et al., Phys. Lett. A 329, 294 (2004).

[13] To our knowledge, the name 'sculpture' was coined by R.M. Serra et al., Phys. Rev. A 62, 043810 (2000).

[14] C. J. Villas-Bôas, et al., Phys. Rev. A 68, 061801(R) (2003); R. M. Serra, et al., quant-ph/0306126.

[15] M. Brune et al., Phys. Rev. Lett. 65, 976 (1990); Phys. Rev. A 45, 5193 (1992).

[16] J. M. C. Malbouisson and B. Baseia, J. Mod. Opt. 46, 2015 (1999).

[17] J. M. Raimond et al., Phys. Rev. Lett. 79, 1964 (1997).

[18] C. C. Gerry, P. L. Knight, Am. J. Phys. 65, 964 (1997).

[19] H.P. Yuen, Phys. Rev. A 132226 (1976).

[20] B.C. Sanders, G.J. Milburn, Phys. Rev. A 39, 694 (1989).

[21] Preparation of squeezed states for running fields remounts to twenty years ago: see Ref. [2].

[22] C.C. Gerry, Phys. Rev. A 59, 4095 (1999).

[23] A.T. Avelar, J.M.C. Malbouisson, and B. Baseia, Physica A 334, 139 (2004).

[24] G. Yeoman and S.M. Barnett, J. Mod. Opt. 40, 1497 (1993).

[25] N. Imoto, H.A. Haus, and Y. Yamamoto, Phys. Rev. A 32, 2287 (1985).

[26] This recursive procedure replicating the apparatus to get a desired state is not a novelty; see, e.g., A.P. Lund et al., quantph/0401001; M. Dakna et al., Phys. Rev. A59 (1999) 1658; XuBo Zou, K. Pahlke, W. Mathis, Phys. Lett. A323, 329 (2004).

[27] A. Imamoglu, Phys. Rev. Lett. 89, 163602 (2002); D.F.V. James and P.G. Kwiat, Phys. Rev. Lett. 89, 183601 (2002).

[28] A. Auffeves et al., Phys. Rev. Lett. 91, 230405 (2003).

[29] D.F. Walls, G.J. Milburn, Phys. Rev. A 31 (1985) 2403; M. Brune et al., Phys. Rev. A 45, 5193 (1992).

[30] B.C. Sanders and G. J. Milburn, Phys. Rev. A 45, 1919 (1992).

[31] H. Schmidt and A. Imamoglu, Opt. Lett. 21, 1936 (1996); J. D. Franson, Phys. Rev. Lett. 78, 3853 (1997); M. D. Lukin and A. Imamoglu, Phys. Rev. Lett. 84, 1419 (2000).

[32] A. Beveratus et al., Eur. Phys. J. D. 18, 191 (2002); C. Santori et al., Nature 419, 594 (2002). 